\title{
Character of magnetic excitations in a quasi-one-dimensional antiferromagnet near the quantum critical points: Impact on magneto-acoustic properties
}

\author{
O. Chiatti, ${ }^{1}$ A. Sytcheva, ${ }^{1}$ J. Wosnitza, ${ }^{1}$ S. Zherlitsyn, ${ }^{1}$ \\ A. A. Zvyagin, ${ }^{2,3}$ V. S. Zapf, ${ }^{4}$ M. Jaime,${ }^{4}$ and A. Paduan-Filho ${ }^{5}$ \\ ${ }^{1}$ Hochfeld-Magnetlabor Dresden (HLD), Forschungszentrum Dresden-Rossendorf, D-01314 Dresden, Germany \\ ${ }^{2}$ Max-Planck-Institut für Physik komplexer Systeme, D-01187 Dresden, Germany \\ ${ }^{3}$ B. Verkin Institute for Low Temperature Physics and Engineering of the NAS of Ukraine, Kharkov, 61103, Ukraine \\ ${ }^{4}$ National High Magnetic Field Laboratory, Los Alamos National Laboratory, Los Alamos, NM 87545, USA \\ ${ }^{5}$ Instituto de Fisica, Universidade de São Paulo, 05315-970 São Paulo, Brazil
}

(Dated: November 15, 2018)

\begin{abstract}
We report results of magneto-acoustic studies in the quantum spin-chain magnet $\mathrm{NiCl}_{2}$ $4 \mathrm{SC}\left(\mathrm{NH}_{2}\right)_{2}$ (DTN) having a field-induced ordered antiferromagnetic (AF) phase. In the vicinity of the quantum critical points (QCPs) the acoustic $c_{33}$ mode manifests a pronounced softening accompanied by energy dissipation of the sound wave. The acoustic anomalies are traced up to $T>T_{N}$, where the thermodynamic properties are determined by fermionic magnetic excitations, the "hallmark" of one-dimensional (1D) spin chains. On the other hand, as established in earlier studies, the AF phase in DTN is governed by bosonic magnetic excitations. Our results suggest the presence of a crossover from a 1D fermionic to a 3D bosonic character of the magnetic excitations in DTN in the vicinity of the QCPs.
\end{abstract}

PACS numbers: $72.55 .+\mathrm{s}, 75.45 .+\mathrm{j}$

The interest in quasi-1D quantum spin systems has grown considerably during the last decade. This is fostered by the progress in preparing materials with welldefined 1D spin subsystems and the possibility of analyzing the experimental data with the help of nonperturbative theories for $1 \mathrm{D}$ models. $\frac{1}{}$ In addition, such systems often manifest quantum phase transitions at $T=0$ which are governed by parameters other than the temperature. True 1D models do not exhibit any longrange order at finite temperatures $\stackrel{1}{\underline{1}}$ Real quasi-1D antiferromagnetic $(\mathrm{AF})$ materials, containing weakly coupled spin chains with gapless spectra of their low-lying excitations, are usually magnetically ordered at low temperatures. At temperatures higher than the Neél temperature, $T_{N}$, but of the order of the exchange constant, these systems behave as quantum spin chains, where any longrange magnetic order is destroyed by enhanced quantum fluctuations. $\frac{1}{\underline{*}}$ One should note that quasi-1D magnets, in which the low-energy eigenstates of their 1D subsystems have spin gaps, usually do not manifest long-range magnetic ordering. $\stackrel{2}{=}$ However, an external magnetic field can close the spin gap, $\Delta$, and for $H>H_{c} \sim \Delta$ a quantum phase transition to a phase with gapless spin excitations takes place. A further increase of the field yields a second quantum phase transition to a spin-polarized phase at $H>H_{s}$. In the spin-polarized phase the low-energy excitations are also gapped. Hence, the magnetically ordered phase can be observed in the field domain where spin excitations are gapless, and the Néel temperature in such systems is field dependent. The magnetic susceptibilities of a quasi-1D spin system in mean-field approximation can be written as

$$
\chi_{\mathbf{q}}^{\alpha}=\frac{\left(\chi_{\mathbf{q}}^{\alpha}\right)^{(1)}(T)}{1-Z J_{\perp}(\mathbf{q})\left(\chi_{\mathbf{q}}^{\alpha}\right)^{(1)}(T)},
$$

where the superscript (1) denotes the susceptibility of one chain, $\alpha=x, y, z, J_{\perp}$ is the weak interchain exchange constant, $Z$ is the coordination number, and $\mathbf{q}$ is the wave vector. The quasi-1D spin system becomes ordered when the denominator becomes zero (which defines $T_{N}(H)$ ).

The low- $T$ thermodynamics of a state with long-range magnetic order is determined by bosonic excitations, magnons. Recently, several groups have observed phenomena in some AF systems that have been interpreted as Bose-Einstein condensation (BEC) of magnons, viz., as a thermodynamically large number of magnons in the same ground state $3, \frac{3,5}{5}$ For quasi-1D spin systems at $T>T_{N}$ the projection of a single spin may have only a limited number of values (e.g., two values for spin- $1 / 2$ systems, three values for spin-1, etc.). That is why thermodynamic properties of, e.g., $\mathrm{AF}$ spin- $1 / 2$ chains are often determined by low-energy eigenstates which be-

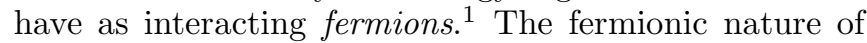
the low-energy excitations of these quantum spin chains with only short-range correlations is related to the limited number of projections of each spin. Very recently it has been shown $\underline{6}^{\underline{-}}$ that the behavior of a spin- 1 spingapped system in magnetic fields close to field-induced quantum critical points (QCP's) can be described by free fermions as well. The fermionic nature of these excitations is also related to the limited number of projection values at each spin-1 site. The fermionic behavior of excitations is characteristic for quantum spin chains with short-range correlations. From this perspective it is very interesting to study the behavior of a spin-gapped quasi1D AF system in the vicinity of $T_{N}$, close to $H_{c}$ and $H_{s}$. Here, low-lying excitations of the quasi-1D system should change their statistical properties from fermionic, at $T>T_{N}(H)$, to bosonic, at $T<T_{N}(H)$. Hence, by varying $H$ and $T$ one may observe features in the same 
spin system characteristic either to fermions or bosons.

One of the best candidates for studying such a crossover in the excitation statistics is the spin-1 system dichloro-tetrakis thiourea-nickel(II), $\mathrm{NiCl}_{2}-4 \mathrm{SC}\left(\mathrm{NH}_{2}\right)_{2}$, known as DTN. Recently, some features in the magnetically ordered phase of DTN at $H \geq H_{c}$ and $T<T_{N}(H)$ were interpreted as BEC of spin degrees of freedom ${ }^{7}$ The bosonic character of the spin excitations in DTN in the magnetically ordered phase has been corroborated and is considered to be a well established fact. In this work, we study magnetic and magneto-acoustic characteristics of DTN near the critical values of $H_{c}$ and $H_{s}$. We show that the behavior of the observed properties outside of the AF phase can be well described by an effective fermionic model of low-lying spin excitations. In this way DTN manifests 1D fermionic character of spin excitations at $T>T_{N}(H)$. This fact, together with previous results, showing bosonic $3 \mathrm{D}$ behavior of magnetic excitations in DTN for $T<T_{N}(H), \underset{7}{ }$ leads us to the conclusion that a crossover from fermionic to bosonic features of the lowlying magnetic excitations takes place at $T_{N}$ near the quantum critical points.

Following Ref. 6] we describe the spin-1 chain at low excitation densities using an effective free-fermion theory with two branches of low-energy states. Two branches are used because the strong single-ion "easy-plane" magnetic anisotropy $D$ observed in $\mathrm{DTN}^{8}$ splits the spin triplet of the spin-gap modes, and makes one of them ineffective at the critical fields ${ }^{6}$ The two fermionic branches have features at $H_{c}$ and $H_{s}$, while for $D \ll T$ the contribution of the third branch is exponentially small and can be neglected in our approximation. Both critical fields are related only to the lowest branch of our model (they correspond to van Hove singularities, connected with two edges of that branch). However, the field dependence is present also in the temperature-dependent factors of both branches. In Fig. 1 the solid line shows the calculated field dependence of the magnetization of the quasi-1D spin system (1D subsystems are considered within this effective free-fermion model) for $T$ slightly above the phase boundary $T_{N}(H)$ (see inset in Fig. 1), where the susceptibility of the quasi-1D system diverges. For comparison we also plot experimental data taken at $T=0.6 \mathrm{~K} .9$ Note that $T_{N}(H)<0.6 \mathrm{~K}$ in the vicinity of the quantum critical points $H_{c}$ and $H_{s}$, since there is a line of phase transitions $T_{N}(H)$ with $T_{N}\left(H_{c}\right)=T_{N}\left(H_{s}\right)=0$ and the system in our model is not in the magnetically ordered phase inside of the interval $H_{c}^{\prime} \leq H \leq H_{s}^{\prime} . H_{c}^{\prime}$ and $H_{s}^{\prime}$ are the critical fields at non-zero $T$. There is a good agreement between the effective free-fermion theory and the experimental data at $H<H_{c}^{\prime}, H>H_{s}^{\prime}$, and near the critical values of $H$. On the other hand, inside of the interval $H_{c}^{\prime}<H<H_{s}^{\prime}$, the real system is ordered, $T_{N}(H)>0.6 \mathrm{~K}$, and our 1D fermionic description cannot be applied.

Ultrasonic investigations are a powerful experimental technique to study various phase transitions and critical phenomena. This technique is well established as an im-

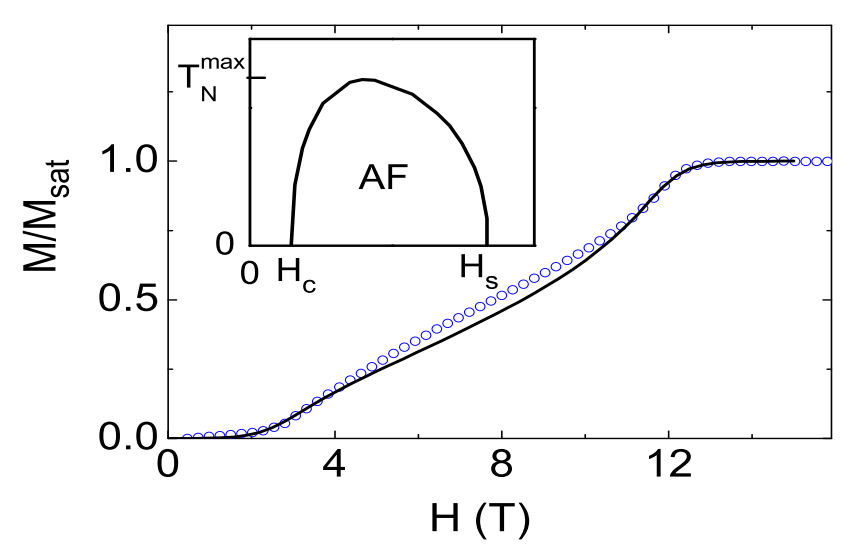

FIG. 1: (Color online) Low-temperature magnetization $(T=$ $0.6 \mathrm{~K})$ of DTN as a function of external magnetic field $H \|[001]$ (circles) $\stackrel{9}{~}^{-T}$ The line is the result of the free-fermion effective theory. The inset sketches the temperature - magnetic field phase diagram of DTN.$^{7}$ The quantum critical points are $H_{c} \approx 2.1 \mathrm{~T}$ and $H_{s} \approx 12.6 \mathrm{~T}$. The maximum temperature of the AF order is $T_{N}^{\max } \approx 1.2 \mathrm{~K}$.

portant tool for the investigation of low-dimensional spin systems 10 Spin-lattice interactions are responsible for the attenuation of acoustic waves and influence the sound velocity in magnetic crystals. These interactions are connected either with a strain modulation of the exchange interactions or with a magnetostrictive coupling of a singleion type ${ }^{10}$ We have performed measurements of the relative change of the sound velocity and attenuation in DTN, using a phase-sensitive detection technique based on a standard pulse-echo method with a set-up similar to the one described in Ref. [10]. DTN has a tetragonal crystallographic symmetry (space group I4) with two formula units in the unit cell. The investigated single crystal has a size of about $2 \times 2 \times 4.1 \mathrm{~mm}^{3}$. Since the as-grown surfaces of the crystal were smooth and parallel, we glued piezoelectric film transducers directly to the surfaces normal to the crystallographic [001] direction, without any additional sample polishing. This geometry corresponds to the longitudinal acoustic $c_{33}$ mode, with propagation direction and polarization along the spin chains. A number of ultrasonic echoes have been detected. The absolute value of the sound velocity at liquid-helium temperature has been determined as $v_{l}=2640 \pm 20 \mathrm{~m} / \mathrm{s}$. Note that the measurement accuracy for a relative change of sound velocity is of the order of $10^{-6}$. The sample-length change is relatively small for the applied temperatures and magnetic fields. 11 Therefore, we did not have to take into account any length-change corrections to the sound velocity. The data have been collected using the ultrasonic signal at $78 \mathrm{MHz}$. The magnetic field was applied along the [001] direction, i.e., parallel to the sound-propagation direction. Figure 2 shows the magnetic-field dependence of the relative change of the sound velocity and attenuation of the $c_{33}$ mode in DTN for $T$ below the maximum of $T_{N}^{\max } \approx 1.2 \mathrm{~K}$. There is a pronounced softening 


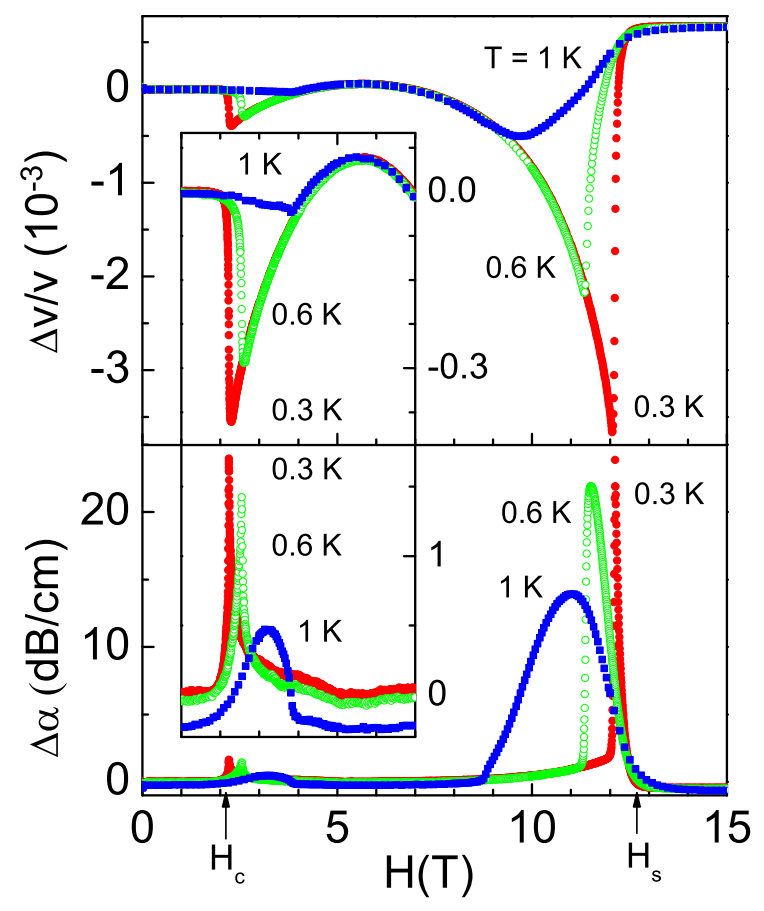

FIG. 2: (Color online) Field dependence of the relative change of the sound velocity (top) and of the sound attenuation (bottom) of the acoustic $c_{33}$ mode in DTN at $T$ below $T_{N}^{\max }$. The magnetic field was applied along the [001] axis. The ultrasonic frequency was $78 \mathrm{MHz}$. The insets show the sound velocity and attenuation in the vicinity of $H_{c}$ in enlarged scale.

of the $c_{33}$ mode in the vicinity of both critical fields, though the anomaly at $H_{s}^{\prime}$ is approximately one order of magnitude larger than that at $H_{c}^{\prime}$. There is a relative increase of $\Delta v / v=7 \times 10^{-4}$ between the sound velocity at $H=0$ and $H>12.6 \mathrm{~T}$, where all spins are polarized. The relative decrease of the sound velocity reaches about $4 \times 10^{-3}$ at $12 \mathrm{~T}$ and $0.3 \mathrm{~K}$. The softening of the $c_{33}$ mode is accompanied by a peak in the sound attenuation. Both the sound-velocity and soundattenuation anomalies become smaller and broader with increasing $T$. The $H$ dependence of the sound velocity in the ordered phase (far from the critical regions) resembles $c$-axis magnetostriction data $\stackrel{11}{11}$ However, the change in the sound velocity cannot be explained by the latticeparameter change, since the length change observed in Ref. 11 is too small. In Fig. 3, we show the field dependence of the sound velocity and attenuation of the $c_{33}$ mode in DTN at various temperatures above $T_{N}^{\max }$. One can see some transformation of the acoustic anomalies by moving from $T<T_{N}^{\max }$ to $T>T_{N}^{\max }$. Here, the softening of the $c_{33}$ mode disappears in the vicinity of $H_{c}$; only a smooth increase in the sound velocity is detected. Close to $H_{s}$ one can still observe a minimum in the sound velocity and a maximum in the sound attenuation, but those anomalies are smaller in amplitude and broader than the corresponding ones measured below $T_{N}^{\max }(H)$

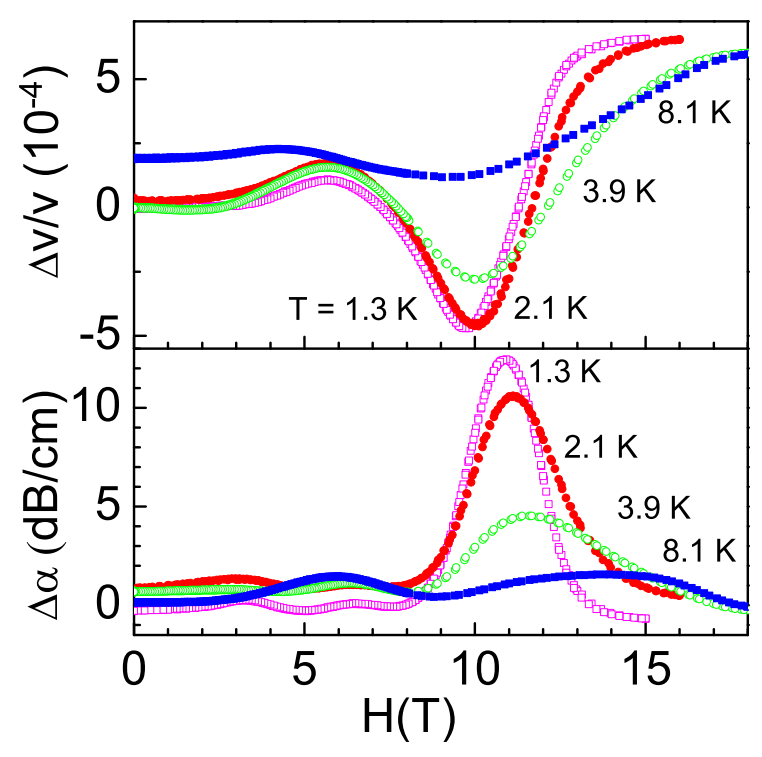

FIG. 3: (Color online) Field dependence of the relative change of the sound velocity (top) and of the sound attenuation (bottom) of the acoustic $c_{33}$ mode in DTN for $T$ above $T_{N}^{\max }$.

(Fig. 2).

In magnetic materials the dominant contribution to the spin-lattice interactions mostly arises from the exchangestriction coupling. In our calculations we assumed that in DTN the spatial dependence of the magnetic anisotropy constant is weaker than the spatial dependence of the exchange integrals. In this case, one can expect that only longitudinal sound waves interact with the spin subsystem. According to Ref. 12, the relative renormalization of the longitudinal sound velocity can be written as $(\Delta v / v)=-\left(A_{1}+A_{2}\right) /\left(N \omega_{\mathbf{k}}\right)^{2}$, where

$$
\begin{aligned}
& A_{1}=2\left|G_{0}^{z}(\mathbf{k})\right|^{2}\left\langle S_{0}^{z}\right\rangle^{2} \chi_{0}^{z}+T \sum_{\mathbf{q}} \sum_{\alpha=x, y, z}\left|G_{\mathbf{q}}^{\alpha}(\mathbf{k})\right|^{2}\left(\chi_{\mathbf{q}}^{\alpha}\right)^{2} \\
& A_{2}=H_{0}^{z}(\mathbf{k})\left\langle S_{0}^{z}\right\rangle^{2}+\frac{T}{2} \sum_{\mathbf{q}} \sum_{\alpha=x, y, z} H_{\mathbf{q}}^{\alpha}(\mathbf{k}) \chi_{\mathbf{q}}^{\alpha}
\end{aligned}
$$

Here, $N$ is the number of spins in the system, $\omega_{\mathbf{k}}=v k$ is the low- $k$ dispersion relation with sound velocity $v$ in the absence of spin-phonon interactions, $\left\langle S_{0}^{z}\right\rangle$ is the average magnetization along the direction of the magnetic field, $\chi_{\mathbf{q}}^{x, y, z}$ are non-uniform magnetic susceptibilities, and the subscript 0 corresponds to $q=0$. In the framework of our effective free-fermion model the temperature and magnetic-field dependence of the uniform susceptibility of one spin chain can be written as

$$
\begin{aligned}
& \left(\chi_{0}^{z}\right)^{(1)}=\frac{8}{\pi T} \int_{H_{c}}^{H_{s}} \frac{x d x}{\sqrt{\left(H_{s}^{2}-x^{2}\right)\left(x^{2}-H_{c}^{2}\right)}} \times \\
& \frac{1+\cosh (H / T) \cosh (x / T)}{[\cosh (H / T)+\cosh (x / T)]^{2}},
\end{aligned}
$$

where we set the units for the effective $g$-factor, Bohr's magneton, and Boltzmann's constant equal to 1. For 


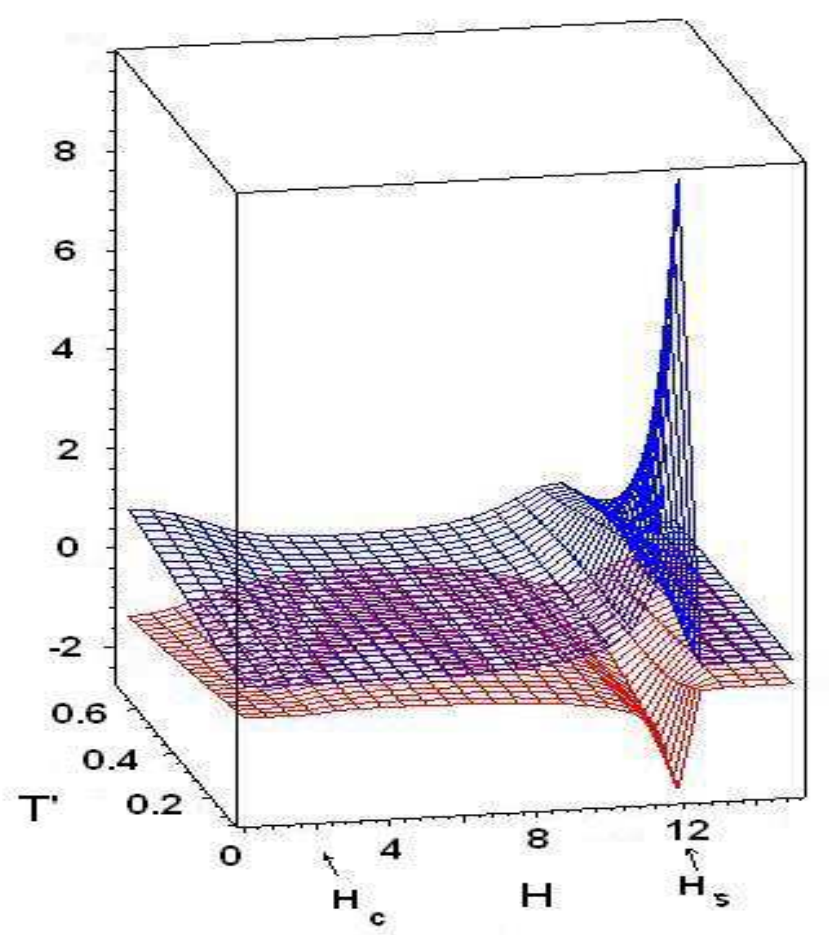

FIG. 4: (Color online) Attenuation (upper surface, blue) and relative change of the velocity (lower surface, red) of the longitudinal sound versus $H$ and $T^{\prime}$, calculated in the framework of the proposed theory (arbitrary units were used for all parameters, see text for details).

spin systems with AF interactions the main contribution to the summation over $\mathbf{q}$ in Eqs. (2) comes from terms with $q=\pi$,

$$
\begin{aligned}
& \left(\chi_{\pi}^{z}\right)^{(1)}=\frac{8}{\pi} \int_{H_{c}}^{H_{s}} \frac{d x}{\sqrt{\left(H_{s}^{2}-x^{2}\right)\left(x^{2}-H_{c}^{2}\right)}} \times \\
& \frac{\sinh (x / T)}{\cosh (H / T)+\cosh (x / T)} .
\end{aligned}
$$

To calculate magnetic susceptibilities of the quasi-1D spin system we use Eqs. (1), (3) and (4).

The renormalization is proportional to the spinphonon coupling constants

$$
\begin{aligned}
& G_{\mathbf{q}}^{\alpha}=\frac{1}{m} \sum_{n} e^{i \mathbf{q} \mathbf{R}_{n m}}\left(e^{i \mathbf{k} \mathbf{R}_{n m}}-1\right) \mathbf{e}_{\mathbf{k}} \frac{\partial J_{m n}^{\alpha}}{\partial \mathbf{R}_{m}} \\
& H_{\mathbf{q}}^{\alpha}=\frac{1}{m} \sum_{n} e^{-i \mathbf{q} \mathbf{R}_{n m}}\left(e^{i \mathbf{k} \mathbf{R}_{n m}}-1\right)\left(e^{-i \mathbf{k} \mathbf{R}_{n m}}-1\right) \times \\
& \mathbf{e}_{\mathbf{k}} \mathbf{e}_{-\mathbf{k}} \frac{\partial^{2} J_{m n}^{\alpha}}{\partial \mathbf{R}_{n} \partial \mathbf{R}_{m}} .
\end{aligned}
$$

Here, $m$ is the mass of the magnetic ion, $J_{m n}^{\alpha}$ denote (anisotropic, generally speaking) exchange integrals, $\mathbf{e}_{\mathbf{k}}$ is the polarization of the phonon with wave vector $\mathbf{k}$, and $\mathbf{R}_{n}$ is the position vector of the $n$-th site ${ }^{12}$ Figure 4 (lower surface) shows the $H$ and $T^{\prime}$ dependence of the relative change of the longitudinal sound velocity of a quasi-
$1 \mathrm{D}$ spin system calculated in the framework of the effective free-fermion model. We fixed $H_{c}$ and $H_{s}$ and used arbitrary units for $T^{\prime}$ in Fig. 4 (they are not equal to the temperatures in the experiment). It is challenging to calculate $\chi_{\mathbf{q}}^{x, y}$ in the framework of the used model. Clearly they have to be smooth functions of $H$ and $T$, except at the line $T_{N}(H) . G_{\mathbf{q}}^{\alpha}(\mathbf{k})$ and $H_{\mathbf{q}}^{\alpha}(\mathbf{k})$ in Eqs. (2) are also unknown for any $\alpha$ (one of the coupling constants can be estimated using Ref. 11). That is why, in order to obtain the results presented in Fig. 4, we used $J_{\perp}(q)=0.18$ from Ref. $8, \chi_{\mathbf{q}=\mathbf{0}}^{z}$ and $\chi_{\mathbf{q}=\pi}^{z}$ multiplied by some (not known) values of the spin-phonon coupling constants, and $H^{z}(\mathbf{k})$ two times smaller than $G^{z}(\mathbf{k})$. The temperature of the divergence in the magnetic susceptibility of the quasi-1D system is generally determined by anisotropic couplings between the spin chains (these couplings are unknown). The divergence at $T_{N}(H)$, which we used in our theory, does not depend on the direction of the order parameter. Such divergences are present in a quasi-1D model, when any component of the magnetic susceptibility (but with different phase-transition temperatures, $\left.T_{N}(H)\right)$ is considered. Even in this approximation our simplified theory reproduces the main features of the experimentally observed behavior. Our model reproduces the pronounced minimum at $H_{s}^{\prime}$, the almost field-independent behavior at $H>H_{s}^{\prime}$ and $H<H_{c}^{\prime}$, the larger value of $\Delta v / v$ for $H>H_{s}^{\prime}$ as compared to $H<H_{c}^{\prime}$, and the maximum (with $\Delta v / v>0$ ) in the interval between $H_{c}^{\prime}$ and $H_{s}^{\prime}$. With increasing $T^{\prime}$ the features near the critical fields become weaker, the same way as it was observed in the experiment (cf. Fig. 2 and Fig. 3). At the phase boundary $T_{N}(H)$ the susceptibility of the quasi-1D system diverges (see above), and our theory predicts very narrow and large peaks at the critical values of $H$ (not shown). Therefore, for the sake of clarity, the curves in Fig. 4 are not plotted starting from $T^{\prime}=0 . T_{N}^{\max }(H)$ in our units is $T^{\prime}=0.02$. Concerning the other values of $\chi_{\mathbf{q}}$ (i.e., $\mathbf{q} \neq 0, \pi$ ) we affirm, as it was discussed above, that their inclusion does not affect the qualitative behaviour of the sound velocity and attenuation. Following Ref. 12 we also calculated the attenuation coefficient for DTN,

$$
\begin{aligned}
& \Delta \alpha\left(\equiv \Delta \alpha_{k}\right)=\frac{1}{N v}\left[2\left|G_{0}^{z}(\mathbf{k})\right|^{2}\left\langle S_{0}^{z}\right\rangle^{2} \chi_{0}^{z} \frac{\gamma_{0}^{z}}{\left(\gamma_{0}^{z}\right)^{2}+\omega_{\mathbf{k}}^{2}}\right. \\
& \left.+T \sum_{\mathbf{q}} \sum_{\alpha=x, y, z}\left|G_{\mathbf{q}}^{\alpha}(\mathbf{k})\right|^{2}\left(\chi_{\mathbf{q}}^{\alpha}\right)^{2} \frac{2 \gamma_{\mathbf{q}}^{\alpha}}{\left(2 \gamma_{\mathbf{q}}^{\alpha}\right)^{2}+\omega_{\mathbf{k}}^{2}}\right],
\end{aligned}
$$

where $\gamma_{\mathbf{q}}^{\alpha}$ are the relaxation rates, which can be approximated by $\gamma_{\mathbf{q}}^{\alpha}=B / T \chi_{\mathbf{q}}^{\alpha}$, where $B$ is a material-dependent constant (see Ref. 12). In our calculations we used the approximation, in which the relaxation rates do not depend on the direction and on the wave vector. The results are also presented in Fig. 4 (upper surface). Here, our theory reproduces also the main features observed in the experimental data: an abrupt increase of the sound attenuation near the saturation field $H_{s}$, and damping with increasing $T^{\prime}$. All these findings demonstrate the important role the fermionic magnetic excitations play in the 
vicinity of the QCPs in DTN.

We also tried to reproduce the observed experimental results using the scaling-like procedure, proposed in Ref. 13. In the framework of that approach we can use $\left(\chi_{\pi}^{x, y}\right)^{(1)}$, which seems more accurate than the use of $\left(\chi_{\pi}^{z}\right)^{(1)}$ only. However, the agreement between the theory and experiment was worse than for our effective freefermion model.

Generally speaking, one could as well use a bosonic, say the Holstein-Primakoff, representation of spin operators $\underline{14}$ for any temperatures in DTN. However, to describe the behavior of spins for $T>T_{N}(H)$ one has to take into account all interactions between these bosons (because the interactions are of the same magnitude as the energy of the free bosons), which is impossible so far. We do not know any other theory, bosonic or fermionic, which can describe the behavior of the magnetization, sound velocity, and attenuation in DTN better than the theory presented here. The situation, e.g., in weakly coupled spin ladders, is very different from the one in DTN, because in our case one cannot consider any of the spin-spin interactions as weak. Also, the use of hardcore bosons for the description of the behavior of DTN for $T>T_{N}(H)$ cannot help because from the viewpoint of their collective behavior they can be regarded as fermions (i.e., only one fermion, or hard-core boson can be in one state). For $T<T_{N}(H)$ in DTN, we definitely cannot use hard-core bosons (e.g., for hard-core bosons BEC is impossible, however, see Ref. 7] for DTN).
The advantage of our fermionic description of DTN for $T>T_{N}(H)$ (and the mentioned bosonic description for $T<T_{N}(H)$ ), compared to the use of only a bosonic description of low-energy spin excitations, is that in our approach both fermions and bosons are basically noninteracting. Hence, they have all features of standard fermions and bosons. For strongly interacting bosons, which is the case for hard-core bosons or the HolsteinPrimakoff representation for $T>T_{N}(H)$, one cannot, strictly speaking, use directly the bosonic character of these excitations. We finally want to note, that in our calculations we never used the symmetry of the wave function, the other difference between fermions and bosons.

In summary, our magnetic and magneto-acoustic studies of the quantum spin-chain magnet $\mathrm{NiCl}_{2}-4 \mathrm{SC}\left(\mathrm{NH}_{2}\right)_{2}$ show that the behavior of the observed properties at $T>$ $T_{N}(H)$ can be well described by an effective 1D fermionic model of low-lying spin excitations. This fact, together with previous results showing the bosonic $3 \mathrm{D}$ behavior of the magnetic excitations in DTN for $T<T_{N}(H) \stackrel{7}{\underline{7}}$ suggests the presence of a crossover from a fermionic to a bosonic character of the magnetic excitations close to the quantum critical points. The fermionic and bosonic nature of the magnetic excitations is related to the shortrange correlations in the spin chains and to the longrange three-dimensional order, respectively.

We thank S. A. Zvyagin for stimulating discussions. A.A.Z. acknowledges the support from the Ukrainian Fundamental Research State Fund (F25.4/13).
1 See, e.g., A. A. Zvyagin Finite Size Effects in Correlated Electron Models: Exact Results, Imperial College Press, London, 2005.

2 See, e.g., L. P. Regnault et al., Phys. Rev. B 53, 5579 (1996).

3 S. E. Sebastian et al., Nature 441, 617 (2006).

4 T. Radu et al., Phys. Rev. Lett. 95, 127202 (2005).

${ }^{5}$ Ch. Rüegg et al., Nature 423, 62 (2003).

6 Y. Maeda, C. Hotta, and M. Oshikawa, Phys. Rev. Lett. 99, 057205 (2007).

7 V. S. Zapf et al., Phys. Rev. Lett. 96, 077204 (2006).

8 S. A. Zvyagin et al., Phys. Rev. Lett. 98, 047205 (2007).
9 A. Paduan-Filho et al., Phys. Rev. B 69, 020405(R) (2004).

10 See, e.g., B. Lüthi, Physical Acoustics in the Solid State, Springer, Berlin, 2005.

11 V. S. Zapf et al., Phys. Rev. B 77, 020404(R) (2008)

12 M. Tachiki and S. Maekawa, Progr. Theor. Phys. 51, 1 (1974)

13 Z. Honda, K. Katsumata, Y.Nishiyama, and I. Harada, Phys. Rev. B 63, 064420 (2001).

14 T. Holstein, and H. Primakoff, Phys. Rev. 581098 (1940). 